\title{
Cytokines as determinants of resistance and pathology in human Schistosoma mansoni infection
}

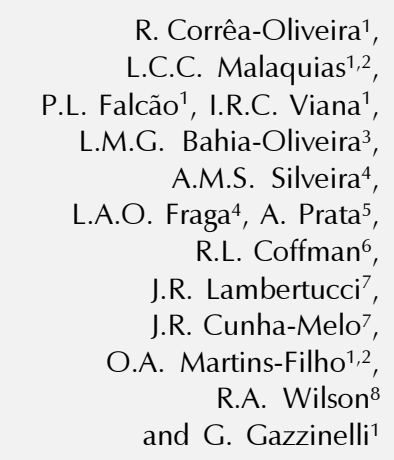

Correspondence

R. Corrêa-Oliveira

Laboratório de Imunologia Celular

e Molecular

Centro de Pesquisas René Rachou FIOCRUZ

Avenida Augusto de Lima, 1715 30190-002 Belo Horizonte, MG Brasil

Fax: 55 (013) 295-3115

Presented at the International Meeting on Cytokines, Angra dos Reis, RJ, Brasil, November 24-28, 1996.

Research supported by the European Commission, INCO-DC Programme, NIH (No. Al 26505), CNPq, FAPEMIG, FINEP-CNPq (PRONEX) and the UNDP/World Bank/WHO Special Programme for Research and Training in Tropical Diseases.

\author{
${ }^{1}$ Laboratório de Imunologia Celular e Molecular, Centro de Pesquisas René Rachou, \\ FIOCRUZ, Belo Horizonte, MG, Brasil \\ ${ }^{2}$ Departamento de Bioquímica e Imunologia, Instituto de Ciências Biológicas, \\ Universidade Federal de MInas Gerais, Belo Horizonte, MG, Brasil \\ ${ }^{3}$ Laboratório de Biologia do Reconhecer, Universidade Estadual do \\ Norte Fluminense, Campos de Goitacazes, Campos, RJ, Brasil \\ ${ }^{4}$ CENBIOS, Universidade do Vale do Rio Doce, Governador Valadares, MG, Brasil \\ ${ }^{5}$ Departamento de Medicina Tropical, Faculdade de Medicina do Triângulo Mineiro, \\ Uberaba, MG, Brasil \\ ${ }^{6}$ DNAX Research Institute, Palo Alto, CA, USA \\ ${ }^{7}$ Departamento de Clínica Médica, Faculdade de Medicina, Universidade Federal de \\ Minas Gerais, Belo Horizonte, MG, Brasil \\ ${ }^{8}$ Department of Biology, University of York, York, United Kingdom
}

\section{Abstract}

The role of different cytokines in the peripheral blood mononuclear cell (PBMC) proliferative response and in in vitro granuloma formation was evaluated in a cross-sectional study with patients with the different clinical forms and phases of Schistosoma mansoni infection, as well as a group of individuals "naturally" resistant to infection named normal endemic (NE). The blockage of IL-4 and IL-5 using anti-IL-4 and anti-IL-5 antibodies significantly reduced the PBMC proliferative response to soluble egg (SEA) and adult worm (SWAP) antigens in acute (ACT), chronic intestinal (INT) and hepatosplenic (HS) patients. Similar results were obtained in the in vitro granuloma formation. Blockage of IL-10 had no significant effect on either assay using PBMC from ACT or HS. In contrast, the addition of anti-IL-10 antibodies to PBMC cultures from INT patients significantly increased the proliferative response to SEA and SWAP as well as the in vitro granuloma formation. Interestingly, association of anti-IL-4 and anti-IL-10 antibodies did not increase the PBMC proliferative response of these patients, suggesting that IL-10 may act by modulating IL-4 and IL-5 secretion. Addition of recombinant IL-10 decreased the proliferative response to undetectable levels when PBMC from patients with the different clinical forms were used. Analysis of IFN- $\gamma$ in the supernatants showed that PBMC from INT patients secreted low levels of IFN- $\gamma$ upon antigenic stimulation. In contrast, PBMC from NE secreted high levels of IFN- $\gamma$. These data suggest that IL-10 is an important cytokine in regulating the immune response and possibly controlling morbidity in human schistosomiasis mansoni, and that the production of IFN- $\gamma$ may be associated with resistance to infection.
Key words

- Schistosoma mansoni

- Human schistosomiasis mansoni

- Cytokines

- IL-10

- IL-4

- IFN- $\gamma$ 


\section{Introduction}

Infection by Schistosoma mansoni (S. mansoni) in man induces multiple cellular and humoral immune responses to the parasite antigens that are believed to determine the outcome of the disease. These responses have been shown to be correlated with the phase (acute vs chronic) and the clinical form of the infection $(1,2)$. Several laboratories have demonstrated that during the development of the disease significant changes in cellular proliferative responses occur. While peripheral blood mononuclear cells (PBMC) from individuals with the acute phase (ACT) show significant in vitro proliferative responses to soluble egg antigens (SEA) and to adult worm antigens (SWAP), the PBMC response of patients with the chronic asymptomatic intestinal (INT) clinical form of the disease to SEA is significantly reduced. This decrease has been suggested to be due to the regulation of the immune response to the parasite eggs trapped in the tissues. Analysis of the cellular immune response of individuals with the severe form of the disease (hepatosplenic schistosomiasis) has demonstrated that those with the compensated form have an increased response to SEA while patients with the decompensated form show a significant decrease in the response to both SEA and SWAP, thus being considered non-responders $(3,4)$.

The identification of immune mechanisms involved in resistance to infection has focused mainly on the analysis of the immune response of patients with the intestinal clinical form of schistosomiasis. The fact that this group presents infection by S. mansoni argues against these individuals having an effective immune response to the invading parasites. With this assumption in mind we have identified a group of patients consisting of individuals who have continuous water contact but no infection. This group was named "normal endemic" (NE) (5) and is assumed to be naturally resistant to infec- tion. We have also included this group in the studies described in this review.

Several laboratories have been working on the identification of the immune mechanisms involved in the development of pathology and resistance to infection or reinfection. Among the most extensively studied immune factors are the anti-schistosome antibodies. Within this context, idiotype/antiidiotype interactions $(6,7)$, adherent cells $(8,9)$, "suppressor cells" (10), immunocomplexes (11) and soluble factors (12) have been demonstrated to play a role in cellular responses. A crucial role of $\mathrm{T}$ cells in the pathology of the infection has been well demonstrated both in experimental models and in man $(13,14)$. Furthermore, an effective immune response capable of partially preventing the infection has been correlated with the activity of antibodies (15) and macrophages (16).

The immunopathology of Schistosoma mansoni infection is characterized by a delayed type inflammatory granulomatous reaction to the parasite eggs trapped in the tissues of the vertebrate host. This immune response can eventually lead to the development of severe hepatic fibrosis, portal hypertension, gastrointestinal hemorrhage, and death (17-19). This response, however, decreases significantly as described above for cellular proliferation and this phenomenon has been named granuloma modulation $(20,21)$. The decrease in granuloma size coincides with the reduction of the symptoms observed during the chronic phase of the infection (22).

In this review we describe the recent findings about the role of cytokines in the development of pathology and resistance to infection. We have performed a cross-sectional study in which patients in the acute phase of the disease, or with the chronic intestinal or hepatosplenic (compensated and decompensated) clinical forms were evaluated. The normal endemic group was also included in an attempt to identify differences 
between these groups of patients that might explain the severe vs mild clinical forms and resistance to infection, both in terms of PBMC proliferative response and in vitro granuloma formation.

The acute phase of schistosomiasis

The limited number of studies on the immune response of the acute phase of schistosomiasis is due to the fact that in endemic areas the occurrence of this clinical form of the disease is very low or negligible. It is also possible that the symptoms are mild and the patient does not suffer any major distress from the infection.

Analysis of the proliferative responses of PBMC from ACT patients in the presence of blocking antibodies to IL-4 and IL-5 demonstrated that anti-IL-4 antibodies significantly reduce the cellular proliferative response to SEA but not to SWAP. Similar results were obtained in the in vitro granuloma assay, further supporting the role of these cytokines in the immune response during the acute phase and in granuloma modulation. These results are in agreement with those previously published by Cheever (18) for murine schistosomiasis, suggesting that IL-4 is an important cytokine in granuloma formation.

IL-10 has been shown to be a major cytokine with down-regulatory activity for both Th1 and Th2 T cell subpopulations (23). Analysis of the role of IL-10 in both PBMC proliferative response (to SEA and SWAP) and in vitro granuloma formation demonstrated that blockage of this cytokine had no significant effect on either assay. These results can be interpreted as a lack of production of this cytokine by PBMC from acute patients or inability of PBMC from these individuals to respond to IL-10. To evaluate these possibilities the effect of addition of recombinant IL-10 to the cultures as well as the effect of soluble IL-10 levels on tissue culture supernatants were determined. The results obtained demonstrated that very low levels of IL-10 are secreted by PBMC from these patients, thus explaining results obtained in the cultures to which antiIL-10 monoclonal antibodies were added. The fact that the addition of recombinant IL10 led to a significant decrease in PBMC proliferative response and in vitro granuloma formation demonstrates that PBMC from patients are responsive to IL-10.

Flow cytometry analysis of the PBMC populations of patients in the acute phase of the disease demonstrated a significant increase in CD4+HLA-DR $+\mathrm{T}$ cells as well as in $\mathrm{CD} 3-\mathrm{CD} 2+(\mathrm{NK})$ cells. These results suggest that significant cell activation occurs during the acute phase of the disease and that this activation may involve IFN- $\gamma$ secretion by NK cells. The observed increase in IFN- $\gamma$ may be important during this phase of the infection for activation of macrophages that in turn will interact with $\mathrm{CD} 4+\mathrm{T}$ cells during their process of differentiation. It is not clear whether an early activation of Th1 cells occurs with a switch to a Th2 response as eggs are laid. The fact that patients in the acute phase of the disease are diagnosed only after they start passing eggs in their stool further impairs this evaluation. Whether IFN- $\gamma$ secretion is also an important factor in the development of the severe acute cases remains to be determined. It is possible that IFN- $\gamma$ secreted by these cells acts via priming of macrophages and $T$ cells, inducing the production of TNF- $\alpha$ acting directly on granuloma formation.

\section{The chronic phase}

of schistosomiasis

Analysis of the role of cytokines in the immune response of individuals in the chronic phase of schistosomiasis was performed as described above for the acute phase. PBMC proliferative response as well as in vitro granuloma formation were also evaluated. The chronic phase of schistosomiasis presents with different clinical forms as de- 
scribed above. To facilitate the understanding of the data, we will present the results for each of the clinical forms of the disease.

\section{Intestinal form of schistosomiasis}

In contrast to the data presented for the patients in the acute phase of the disease, the addition of anti-IL-10 monoclonal antibodies to PBMC cultures from INT individuals induced a significant increase in the proliferative response and in in vitro granuloma size. This is an important observation since it has been well demonstrated that these patients, in contrast to the ACT individuals, have a significant decrease in anti-SEA response and in vitro granuloma size. Anti-IL10 monoclonal antibodies also had a significant effect on the PBMC response of INT individuals to SWAP which, although less dramatic, was significant, again contrasting with the data obtained in the assays with PBMC from ACT patients. To further investigate the role of different cytokines, anti-IL4 and anti-IL-5 monoclonal antibodies were added to the cultures. Addition of anti-IL-4 but not anti-IL-5 antibodies leads to a decrease in both PBMC proliferative response and in vitro granuloma formation. Due to the inhibitory effect of IL-4 blockage, we combined anti-IL-10 antibodies and anti-IL-4 or anti-IL-10 and anti-IL-5 in the cultures from the same patients. The data demonstrate that the increase in the PBMC proliferative response and in in vitro granuloma formation observed by the blockage of IL-10 is readily reversed by the addition of monoclonal antibodies to either IL-4 or IL-5. These data suggest that the increase observed in the cultures with anti-IL-10 antibodies may be due to the blockage of the regulatory effect of IL-10, allowing the secretion of both IL-4 and IL-5 and a consequent increase in cellular proliferation. These data also suggest that during the chronic INT phase of the disease the response is mainly Th2 mediated, also suggesting that the $\mathrm{Th} 2$ response has an im- portant regulatory role in man. It is important to mention that the addition of recombinant IL-10 to the cultures decreased the responses to undetectable levels as observed in the assays with PBMC from ACT individuals.

To investigate the role of a Th1 response of these patients, PBMC proliferative responses, were evaluated in the presence of monoclonal antibodies to IFN- $\gamma$. The data obtained demonstrate that the response to both SEA and SWAP is drastically reduced by the blockage of IFN- $\gamma$. Although the mechanism involved in this decrease is still obscure, we speculate that IFN- $\gamma$ may have an important effect on the antigen-presenting cells and when its activity is blocked these cells may not present antigens to the $T$ cells, blocking the expression of costimulatory molecules such as B7 and CD28. Evaluation of the levels of IFN- $\gamma$ secretion in the supernatants of PBMC cultures demonstrated that very few cells from these patients secrete low to undetectable levels of this cytokine. We have further demonstrated that this is a common feature of chronic schistosomiasis.

Taken together, these data suggest that both Th1 and Th2 cytokines may have important effects on the $T$ cell responses of INT individuals. At this stage it is still premature to attempt to determine what exactly is the role of each cytokine and also whether they are determinants of disease. However, if we compare the data obtained with PBMC from ACT with those of PBMC from INT, the role of IL-10 in the down-modulation of the immune response in chronic asymptomatic schistosomiasis becomes evident, suggesting a possible role of this cytokine in controlling morbidity.

\section{Hepatosplenic form of schistosomiasis}

The severe form of schistosomiasis (hepatosplenic, HS) is defined clinically by the enlargement of spleen and liver. Immunologically it has been clearly demonstrated 
that these patients have distinct in vitro cellular proliferative responses (3) as well as in vitro granuloma formation (Falcão PL, Malaquias LCC, Silveira MAS, Martins-Filho AO, Passos VMA, Prata A, Gazzinelli G, Coffman R and Corrêa-Oliveira R, unpublished data) as described in the Introduction. Along the same lines of the experiments described above, we have evaluated the role of different cytokines in the PBMC proliferative response and the in vitro granuloma formation. The results obtained with cells from HS patients were similar to those of ACT, where blockage of IL-4 caused a significant decrease in theproliferative response and in the in vitro granuloma formation. However, addition of anti-IL-10 monoclonal antibodies to the cultures had no significant effect on the cellular responses, although the addition of recombinant IL-10 readily decreased the already low responses of PBMC from these patients to the parasite antigens. These results are consistent with the proposed important role of IL-10 in the modulation of the immune responses of these individuals and consequently in the control of morbidity.

Analysis of IFN- $\gamma$ secretion in supernatants of tissue cultures demonstrated that, as described for the INT group, very low to undetectable levels of this cytokine are secreted in PBMC cultures stimulated with the parasite antigens (24).

\section{Normal endemic individuals}

Normal endemic individuals (NE) were first described by our laboratory (5). These endemic individuals were characterized based on their high anti-schistosome antigen response and the lack of infection, determined by the absence of S. mansoni eggs in their stool. Furthermore, these individuals also have continuous water contact and no previous history of drug treatment for schistosomiasis. In our initial studies we evaluated the levels of IFN- $\gamma$ secretion by PBMC cultured in the presence of schistosome antigens. The results clearly demonstrate that PBMC from NE patients secreted high levels of this cytokine in response to all antigenic preparations. These results contrast significantly with those observed in PBMC cultures from patients in the chronic phase of the disease. Furthermore, we demonstrated that IFN- $\gamma$ secretion correlates with resistance to infection, supporting a role of the Th1 response in immunity to S. mansoni (25).

It has been well documented that IgE responses to SWAP can be correlated with the development of resistance to reinfection after treatment. $\operatorname{IgE}$ antibodies are an importantindicator of Th2 type response and therefore could be used as an indirect measure. We evaluated the anti-SWAP antibody responses of these patients together with those of the treated non-reinfected individuals. The results did not show any significant difference in the anti-SWAP IgE responses between the NE and infected patients while those treated and resistant to reinfection had a significant increase in this antibody level. The anti-IgE results with sera from treated resistant individuals are in agreement with those previously published (26-31). The most interesting data were obtained when sera from these individuals were used in IgE antischistosomula tegumental antigen assays. The NE patients had significantly higher levels of $\operatorname{IgE}$ antibodies to these antigens when compared to the treated group resistant to reinfection. These data suggest that both the Th1 and Th2 responses are effective in the elimination of S. mansoni infection and that the mechanism of resistance may be multifactorial. These results, although apparently conflicting, suggest that an effective immune response to the invading parasite may be compartmentalized.

\section{Final comments}

The data presented in this review are an initial analysis of the role of Th1 and Th2 
cytokines in the development of resistance and pathology to Schistosoma mansoni infection in man. Due to the fact that these studies were performed in an endemic population, we carried out a cross-sectional study including all clinical forms and phases of the disease as well as a group of individuals with "natural" resistance to infection.

The studies described in this review allowed us to speculate that IL-10 plays a major role in the regulation of human immune response to $S$. mansoni infection. Furthermore, our data suggest that IL-10 is the major cytokine involved in the control of morbidity in human schistosomiasis.
The NE population constitutes a very important group of individuals who, although living in endemic areas with all the features described above, do not get infected. Analysis of the immune response of these individuals is highly relevant for the understanding of immune effector mechanisms involved in the elimination of S. mansoni infection. Post-treatment responses are not similar to those of NE, again suggesting differences in immune protective mechanisms developed "naturally"vs those acquired after drug treatment. Studies on this population will continue and will be undoubtedly relevant for the development of an effective vaccine.

\section{References}

1. Ottesen EA, Hiatt RA, Cheever AW, Sotomayor ZR \& Neva F (1978). The acquisition and loss of antigen-specific cellular immune responsiveness in acute and chronic schistosomiasis in man. Clinical and Experimental Immunology, 33: 38-47.

2. Nash TE, Garcia-Coyo C, Ruiz-Tiben E, Nazario-Lopez HA, Vasquez G \& TorresBorges A (1983). Differentiation of acute and chronic schistosomiasis by antibody responses to specific schistosome antigens. American Journal of Tropical Medicine and Hygiene, 32: 776-784

3. Colley DG, Garcia AA, Lambertucci JR, Parra JC, Katz N, Rocha RS \& Gazzinelli G (1986). Immune response during human schistosomiasis mansoni. XII. Differential responsiveness in patients with hepatosplenic disease. American Journal of Tropical Medicine and Hygiene, 35: 793802.

4. Gazzinelli G, Montesano MA, CorrêaOliveira R, Lima MS, Katz N, Rocha RS \& Colley DG (1987). Immune responses in different groups of schistosomiasis patients. Memórias do Instituto Oswaldo Cruz, 82 (Suppl IV): 95-100

5. Corrêa-Oliveira R, Pearce EJ, Oliveira GC, Golgher DB, Katz N, Bahia LMG, Carvalho OS, Gazzinelli G \& Sher A (1989). The human immune response to defined immunogens of Schistosoma mansoni: elevated antibody levels to paramyosin in stool-negative individuals from two endemic areas in Brazil. Transactions of the Royal Society of Tropical Medicine and Hygiene, 83: 1205-1211.
6. Lima MS, Gazzinelli G, Nascimento E, Parra JC, Montesano MA \& Colley DG (1986). Immune responses during human schistosomiasis mansoni. Evidence of anti-idiotypic $T$ lymphocyte responsiveness. Journal of Clinical Investigation, 78: 983-988.

7. Montesano MA, Lima MS, Corrêa-Oliveira R, Gazzinelli G \& Colley DG (1989). Immune response during human schistosomiasis mansoni. XVI. Idiotypic differences in antibody from patients with different clinical forms of infection. Journal of Immunology, 142: 2501-2506.

8. Ottesen EA (1979). Modulation of the host response in human schistosomiasis. I. Adherent suppressor cells inhibit lymphocyte proliferative responses to parasite antigens. Journal of Immunology, 123: 1639-1644.

9. Todd CW, Goodgame RW \& Colley DG (1979). Immune responses during human schistosomiasis. V. Suppression of schistosome antigen-specific lymphocyte blastogenesis by adherent/phagocytic cells. Journal of Immunology, 122: 1440-1446.

10. Colley DG, Lewis FA \& Goodgame RW (1978). Immune responses during human schistosomiasis. IV. Induction of suppressor cell activity by schistosome antigen preparation and concanavalin A. Journal of Immunology, 120: 1225-1232.

11. Kamal KA \& Higashi GI (1982). Suppression of mitogen induced lymphocyte transformation by plasma from patients with hepatosplenic schistosomiasis mansoni: role of immune complexes. Parasite
Immunology, 4: 283-289.

12. Colley DG, Hieny SE, Bartholomew R \& Cook JA (1977). Immune response during human schistosomiasis. Regulatory effect of patient sera on human lymphocyte blastogenic responses to schistosome antigen preparations. American Journal of Tropical Medicine and Hygiene, 26: 917-925.

13. Buchanan RD, Fine DP \& Colley DG (1973). Schistosoma mansoni infections in mice depleted of thymus-dependent lymphocytes. II. Pathology and altered pathogenesis. American Journal of Pathology, 71: 207-214.

14. Raso $P$, Bernardes RC, Tafuri WL \& Neves J (1978). As dimensões do granuloma causado pelos ovos do Schistosoma mansoni no fígado. Revista da Sociedade Brasileira de Medicina Tropical, 12: 1-6.

15. Capron A, Dessain JP, Capron M \& Bazin $H$ (1975). Specific IgE antibodies in immune adherence of normal macrophage to Schistosoma mansoni schistosomules. Nature, 253: 474-475.

16. James SL, Sher A, Lazidins JK \& Meltzer MS (1982). Macrophages as effector cells of protective immunity in murine schistosomiasis. II: Killing of newly transformed schistosomula in vitro by macrophages activated as a consequence of Schistosoma mansoni infection. Journal of Immunology, 128: 1535-1540.

17. Jordan P \& Webbe J (1982). Schistosomiasis. Heinemann, London.

18. Cheever AW (1993). Schistosomiasis: infection versus disease and hypersensitivity versus immunity. American Journal of 
Pathology, 142: 699-702.

19. Smithers SR \& Doendhoff MJ (1982). Schistosomiasis. In: Cohen S \& Warren KS (Editors), Immunology of Parasitic Diseases. Blackwell Scientific, Oxford.

20. Andrade ZA \& Warren KS (1964). Mild prolonged schistosomiasis in mice: alterations in host response with time and development of portal fibrosis. Transactions of the Royal Society of Tropical Medicine and Hygiene, 58: 53-57.

21. Boros DL, Pelly RP \& Warren KS (1975). Spontaneous modulation of granulomatous hypersensitivity in schistosomiasis mansoni. Journal of Immunology, 114: 1437-1441.

22. Raso P \& Neves J (1965). Contribuição ao conhecimento do quadro anatômico do fígado na forma toxêmica da esquistossomose mansoni através de punçõesbiopsias. Anais da Faculdade de Medicina da Universidade Federal de Minas Gerais, 22: $147-165$.

23. Mosmann TR \& Moore KW (1991). The role of IL-10 in crossregulation of Th1 and Th2 response. Immunoparasitology Today, 12: A49-A53.

24. Bahia-Oliveira LMG, Gazzinelli G, EloiSantos SM, Cunha-Melo JR, AlvesOliveira LF, Silveira AMS, Viana IRC,
Carmo J, Souza A \& Corrêa-Oliveira R (1992). Differential cellular reactivity to adult worm antigens of patients with different clinical forms of schistosomiasis mansoni. Transactions of the Royal Society of Tropical Medicine and Hygiene, 86: 57-61.

25. Viana IRC, Sher A, Carvalho OS, Massara CL, Eloi-Santos SM, Pearce EJ, Colley DG, Gazzinelli G \& Corrêa-Oliveira R (1994). Interferon-gamma production by peripheral blood mononuclear cells from residents of an area endemic for Schistosoma mansoni. Transactions of the Royal Society of Tropical Medicine and Hygiene, 88: 466-470.

26. Dessain JP, Capron M, Bout D \& Capron A (1975). Quantitative determination of specific lgE level in patients with schistosomiasis (S. mansoni or S. haematobium). Clinical and Experimental Immunology, 20: 427-436.

27. Butterworth $A E$, Capron $M$, Cordingley JS, Dalton PR, Dunne DW, Kariuki HC, Kimani G, Koech D, Mugambi M, Ouma $\mathrm{JH}$, Prentice MA, Richardson BA, ArapSiongok TK \& Taylor DW (1985). Immunity after treatment of human schistosomiasis mansoni. II. Identification of resistant individuals, and analysis of their im- mune response. Transactions of the Royal Society of Tropical Medicine and Hygiene, 79: 393-408.

28. Hagan P, Blumenthal UJ, Dunne D, Simpson AJG \& Wilkins HA (1991). Human $\lg E$, IgG4 and resistance to reinfection with Schistosoma haematobium. $\mathrm{Na}$ ture, 349: 243-245.

29. Rihet $P$, Demeure $C E$, Bourgois $A$, Prata $A$ \& Dessein AJ (1991). Evidence for an association between human resistance to Schistosoma mansoni and high anti-larval IgE levels. European Journal of Immunology, 21: 2679-2689.

30. Dunne DW, Butterworth AE, Fulford AJC Kariuki HC, Langley JG, Ouma JH, Capron A, Pierce RJ \& Sturrock RF (1992). Immunity after treatment of human schistosomiasis: association between IgE antibodies to adult worm antigens and resistance to reinfection. European Journal of Immunology, 22: 1483-1494.

31. Viana IRC, Corrêa-Oliveira R, Carvalho OS, Massara CL, Colosimo E, Colley DG \& Gazzinelli G (1995). Comparison of antibody isotype responses to Schistosoma mansoni antigens by infected and putative resistant individuals living in an endemic area. Parasite Immunology, 17: 297-304. 